OPEN ACCESS

Edited by:

Oswin Grollmuss,

Université Paris-Sud, France

Reviewed by:

Xin Tian,

Shaanxi Provincial Hospital of Traditional Chinese Medicine, China

Michiel Dalinghaus,

Erasmus Medical Center, Netherlands

*Correspondence:

Mei Jin

jinmeiaz2019@126.com

†These authors have contributed equally to this work and share first authorship

Specialty section

This article was submitted to

Pediatric Cardiology,

a section of the journal

Frontiers in Cardiovascular Medicine

Received: 10 July 2020

Accepted: 11 February 2021

Published: 02 March 2021

Citation:

Chen L, Duan H, Li X, Yang Z, Jiao M,

Sun K and Jin M (2021) The Causes of Chest Pain in Children and the Criteria for Targeted Myocardial Enzyme Testing in Identifying the Causes of Chest Pain in Children.

Front. Cardiovasc. Med. 8:582129. doi: 10.3389/fcvm.2021.582129

\section{The Causes of Chest Pain in Children and the Criteria for Targeted Myocardial Enzyme Testing in Identifying the Causes of Chest Pain in Children}

\author{
Li Chen ${ }^{1 \dagger}$, Hongzhou Duan ${ }^{2 t}$, Xiaoyan $\mathrm{Li}^{3}$, Zuozhen Yang ${ }^{4}$, Meng Jiao ${ }^{1}$, Kangtai Sun ${ }^{5}$ and \\ Mei $\operatorname{Jin}^{1 *}$ \\ ${ }^{1}$ Department of Pediatric Cardiology, Beijing Anzhen Hospital, Capital Medical University, Beijing, China, ${ }^{2}$ Department of \\ Neurosurgery, Peking University First Hospital, Beijing, China, ${ }^{3}$ Beijing Institute of Heart, Lung and Blood Vessel Diseases, \\ Capital Medical University, Beijing, China, ${ }^{4}$ Ministry of Education, Laboratory of Biosystem Homeostasis and Protection, \\ College of Life Sciences, Zhejiang University, Hangzhou, China, ${ }^{5}$ Ministry of Science and Technology of the People's \\ Republic of China, Beijing, China
}

\begin{abstract}
Aims: Chest pain is a common complaint at pediatric cardiology clinics and often leads to an extensive cardiac evaluation. In this study, we analyzed the causes of chest pain in Chinese children and developed diagnostic procedures and criteria for targeted myocardial enzyme testing.

Methods and Results: We retrospectively analyzed the clinical data of patients aged below 18 years visiting our hospital for chest pain between 2005 and 2019. Based on auxiliary exams and clinical diagnosis, we developed diagnostic procedures and criteria for targeted myocardial enzyme testing in children with chest pain. A total of 7,251 children were included in this study. The chest pain was of cardiac origin in 581 patients (8.0\%). The incidence of non-cardiac chest pain was significantly higher in the preschool group and the school-age group than in the adolescent group (93.5 vs. 93.8 vs. $90.3 \%$, $P<0.05)$. Among children with cardiac chest pain, the most common concomitant symptom was chest tightness (67.0\%). Myocardial enzyme testing was performed in 5,408 patients and was abnormal in 453 patients. We developed a diagnostic procedure and criteria for targeted myocardial enzyme testing using pertinent history, physical examination, and ECG findings or UCG finding. Applying the diagnostic procedure and criteria could lead to the reduction in myocardial enzyme testing while still capturing all cardiac diagnoses.
\end{abstract}

Conclusion: In children, chest pain is mostly benign and rarely cardiac. During diagnosis, targeted myocardial enzyme testing based on medical history and physical examination can effectively reduce resource use.

Keywords: chest pain, children, cardiac, myocardial enzymes, diagnostic procedure 


\section{INTRODUCTION}

Chest pain is a common complaint at pediatric clinics, accounting for $\sim 6$ per 1,000 visits at pediatric emergency room (1), as well as $\sim 1$ per 40 visits at pediatric clinics in the UK (2), To date, no large studies have been conducted to investigate this topic in Chinese children. In adults, chest pain is often caused by severe cardiovascular diseases, while in children, chest pain is mostly benign and rarely cardiac (3-11). In China, however, most parents believe that chest pain in children is cardiac and life-threatening. So most parents bring their child who has chest pain to an emergency room or see a pediatric cardiologist for comprehensive cardiac exams, which affects the child's life and schoolwork and increases the costs to their families. Therefore, it is important to investigate common causes of chest pain in children and develop diagnostic procedures in order to reduce stress to children and their families.

Collins et al. (12), Harahsheh et al. (13), and Etuwewe et al. (14) proposed evaluation methods and procedures for chest pain in children (12-14). In 2011, Friedman et al. (15) investigated the use of echocardiography (UCG) for the diagnosis of chest pain in children and developed diagnostic and treatment procedures based on medical history, physical examination, and electrocardiogram (ECG). Myocardial enzyme tests are currently widely used for the diagnosis of many heart diseases. To date, no studies have investigated targeted cardiac enzyme testing for the diagnosis of chest pain in children. It is important to develop the criteria for targeted myocardial enzyme testing in children with potential cardiac chest pain in order to reduce the economic burden on their families and reduce resource use. In this study, we analyzed the common causes of chest pain in children and the pattern of clinical visits and developed diagnostic procedures and criteria for targeted myocardial enzyme testing.

\section{MATERIALS AND METHODS}

\section{Case Selection}

We retrospectively analyzed the clinical data of children aged 18 years or below who visited Beijing Anzhen Hospital for chest pain between January 1, 2005 and December 31, 2019. The diagnosis was coded based on the 10th revision of the International Statistical Classification of Diseases and Related Health Problems (ICD-10). Patients with incomplete clinical data or known history of heart disease were excluded from this study. We identified patients on the basis of ICD-10 billing codes for chest pain. Our study complies with the Declaration of Helsinki, and the institutional review board for clinical research at Beijing Anzhen Hospital approved the use of patient medical records for this retrospective review.

We analyzed the demographics, history of the present illness, past medical history, family history, concomitant symptoms, clinical department for initial visit, and auxiliary exams. Based on criteria that were applied in retrospect, chest pain was divided into cardiac or non-cardiac $(3-7,9-13,15,16)$ (Tables 1, 2).
TABLE 1 | Cardiac causes of chest pain $(3-7,9-13,15,16)$.

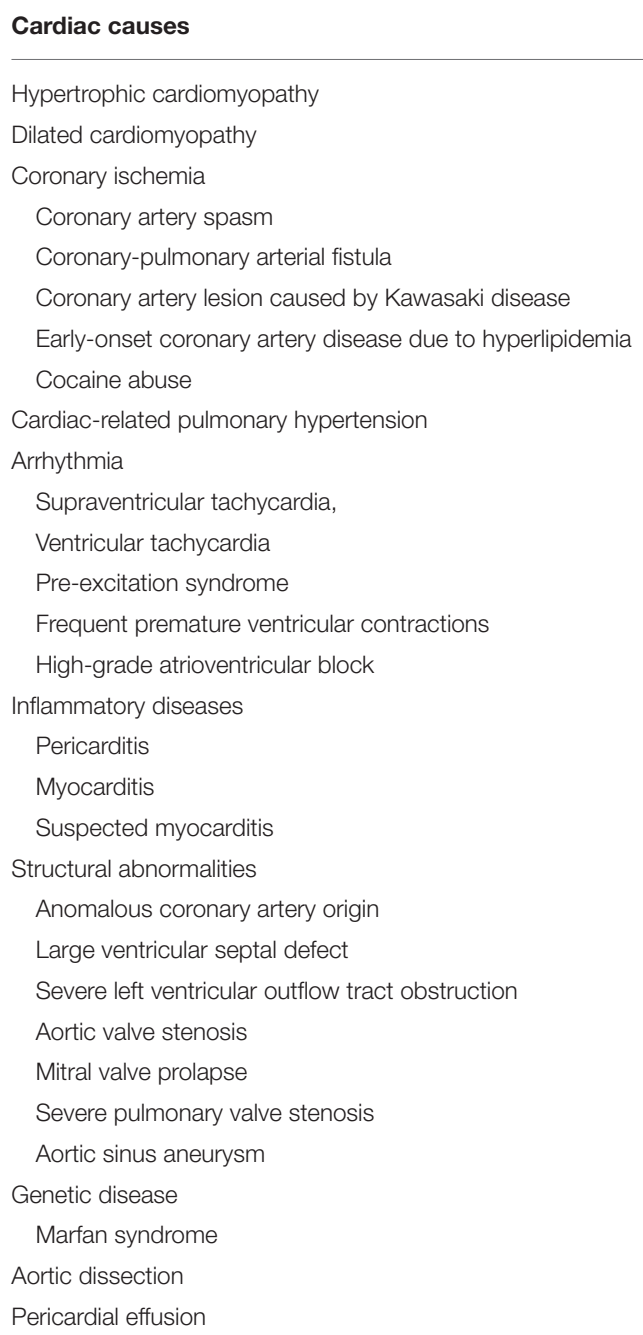

We analyzed the concomitant symptoms in cardiac and non-cardiac patients. Moreover, based on patient age at the initial visit, we divided the patients into the preschool group (3 years $\leq$ age $\leq 6$ years), the school-age group (6 years $<$ age $\leq 12$ years), and the adolescent group (12 years $<$ age $\leq 18$ years). We analyzed the sex composition and the causes of chest pain in each group. Positive history of past illnesses included systemic lupus erythematous, juvenile rheumatoid arthritis, carnitine deficiency, and congenital adrenal insufficiency. Positive family history included sudden or unexplained death of a first-degree relative, severe familial hypercholesterolemia, pulmonary arterial hypertension, Marfan syndrome, and cardiomyopathy. Positive physical examination results included pathological heart murmur, pericardial friction rubs, abnormal second heart sounds, distant heart sounds, arrhythmia, tachycardia, bradycardia, hepatomegaly, pulsus paradoxus, edema, fever, shortness of breath, abnormal breath 
TABLE 2 | Non-cardiac causes of chest pain.

\begin{tabular}{|c|c|}
\hline Non-cardiac causes & Included diseases \\
\hline \multirow[t]{6}{*}{ Musculoskeletal } & Costochondritis \\
\hline & Precordial catch syndrome \\
\hline & Muscle strain \\
\hline & Trauma \\
\hline & Straight-back syndrome \\
\hline & Slipping rib syndrome \\
\hline \multirow[t]{13}{*}{ Respiratory } & Asthma \\
\hline & Pneumonia \\
\hline & Bronchitis \\
\hline & Pleuritis \\
\hline & Acute asthmatic bronchitis \\
\hline & Chronic cough \\
\hline & Emphysema \\
\hline & Mediastinal emphysema \\
\hline & Pulmonary bullae \\
\hline & Pulmonary tuberculosis \\
\hline & Pleural effusion \\
\hline & Pulmonary embolus \\
\hline & Pneumothorax \\
\hline \multirow[t]{8}{*}{ Gastrointestinal } & Gastroesophageal reflux disorder \\
\hline & Esophagitis \\
\hline & Gastritis \\
\hline & Pancreatitis \\
\hline & Esophageal hiatal hernia \\
\hline & Constipation \\
\hline & Gastric ulcer \\
\hline & Biliary disease \\
\hline \multirow[t]{4}{*}{ Psychogenic } & Conversion disorder \\
\hline & Depression \\
\hline & Hysteria-like attacks \\
\hline & Panic/anxiety attack \\
\hline \multirow[t]{5}{*}{ Others } & Skin infection \\
\hline & Dysmenorrhea \\
\hline & Poor wound healing \\
\hline & Breast disease \\
\hline & Acute chest syndrome in sickle cell disease \\
\hline
\end{tabular}

Idiopathic factors

sounds in both lungs, and abnormal muscle strength or muscle tone.

\section{Interpretation of Clinical Auxiliary Exams and Clinical Diagnosis}

We retrieved and analyzed ECG reports from clinical medical records. ECG abnormalities included deviation of the electrical axis, pathological ST-segment or T-wave changes, pathological Q wave, ventricular hypertrophy, atrial enlargement, supraventricular tachycardia, frequent premature ventricular contractions, pre-excitation syndrome, and high-grade atrioventricular block.
UCG, Holter test results, and chest X-ray were analyzed based on reports.

The myocardial enzyme panel included creatine kinase, creatine kinase-myocardial band, lactate dehydrogenase, cardiac troponin I, and myoglobin, which were analyzed based on laboratory reports.

We applied the criteria in retrospect to diagnose the causes of chest pain in children. The clinical diagnostic criteria of myocarditis and suspected myocarditis were listed in Table 3.

\section{Diagnostic Procedures and Criteria for Targeted Myocardial Enzyme Testing for Chest Pain in Children}

We developed diagnostic procedures and criteria for targeted myocardial enzyme testing in children with chest pain based on this retrospective study and the diagnostic procedures for chest pain in children from literature reports $(10,15,17-19)$. In the first step of the diagnostic procedure, we should get detailed history and physical examination results. For the signs and symptoms, we should ask the patients or their parents/guardian that if they have chest tightness, fever, cough, stomach ache, abnormal mental state or palpitations associated with chest pain, syncope during activity, chest pain during exercise, history of cardiac surgery or interventions, complex congenital heart defects, Marfan syndrome, children with on specific cardiac drugs heart transplant, history of Kawasaki disease, history of drug abuse, significant family history of arrhythmias, sudden death in young adulthood, genetic disorders linked with arrhythmias e.g., Long QT syndromes or Brugada syndrome, first-degree relatives have familial hypercholesterolemia, cardiomyopathy and pulmonary hypertension. About physical examination, we should know if they have fever, abnormal breath sounds, pathologic murmur, abnormal heart sounds, hypoxia, peripheral edema, hepatomegaly, significant tachycardia or irregular rhythm. In the second step of the diagnostic procedure, if the patients have chest pain with the following history of the present illness, past medical history, family history and physical examination (History of present illness include post-exercise syncope, chest pain following strenuous exercise or accompanied by chest tightness; past medical history include cardiac surgery or intervention, complex congenital heart disease, Marfan syndrome, Kawasaki disease, drug abuse, and connective tissue disease; family history include hereditary arrhythmia, pulmonary arterial hypertension, and cardiomyopathy, sudden or unexplained death of first-degree relatives under 35 years old, and familial hypercholesterolemia among first-degree relatives; physical examination include pathological heart murmur, abnormal heart sounds, tachycardia, arrhythmia, transcutaneous oxygen saturation, peripheral edema, and hepatomegaly), they should be referred to pediatric cardiologist for diagnosis and treatment. And other patients will be assigned to different departments for diagnosis and treatment. In the third step, doctors from different department will carry out detailed physical examination and necessary auxiliary examination for patients. Based on the results of auxiliary examinations, the doctors will make diagnosis and give treatment for patients. In the 
TABLE 3 | The clinical diagnostic criteria of myocarditis and suspected myocarditis (16).

\begin{tabular}{ll}
\hline $\begin{array}{l}\text { Diagnostic } \\
\text { criteria }\end{array}$ & Contents \\
\hline $\begin{array}{l}\text { The major } \\
\text { criteria }\end{array}$ & $\begin{array}{l}\text { 1. } \\
\text { Syndrome } \\
\text { 2. Cardiomegaly }\end{array}$ \\
3. Cardiac troponin T or I (cTnT/cTnl) or creatinekinase-MB \\
(CK-MB) increased with dynamic changes. \\
4. Significant ECG changes (ECG or Holter)
\end{tabular}

\# Significant ECG changes included ST-T changes in two or more main leads (I, II, aVF $V 5)$ with $R$ wave as the main component lasted for more than 4 days with dynamic changes. Newly found sinus and atrioventricular block, complete right or left bundle branch block, sinus arrest, rhythmic, paired, polymorphic or multi-source premature contraction, non-atrioventricular node and atrioventricular fold ectopic tachycardia, atrial flutter, atrial fibrillation, ventricular flutter, ventricular fibrillation, QRS low voltage (except newborns), abnormal $Q$ wave, etc.

*Typical myocarditis manifestations of CMR refers to at least two of the following three items: (1) myocardial edema: T2 weighted images show localized or diffuse hyperintensity; (2) myocardial congestion and capillary leakage: T1 weighted images show early gadolinium enhancement; (3) myocardial necrosis and fibrosis: T1 weighted images show at least one localized late delayed gadolinium enhancement in non-ischemic areas. \#\# In the three part of minor criteria, if serum $\mathrm{LDH}, \alpha-H B D H$ or AST increased, meanwhile cTnl, cTnT or CK-MB also increased, then they are only regarded as the main indexes, and the important indexes are not calculated repeatedly.

Myocarditis can be diagnosed clinically if there are more than three major criteria, or two major criteria plus three minor criteria, and other diseases are excluded.

Suspected myocarditis can be clinically diagnosed if there are two major criteria, or 1 major criteria plus two minor criteria, or more than three minor criteria, and other diseases are excluded.

In the diagnostic criteria, other diseases that should be excluded include: coronary artery disease, congenital heart disease, high altitude heart disease and metabolic diseases (such as hyperthyroidism and other inherited metabolic diseases), cardiomyopathy, congenital atrioventricular block, congenital complete right or left bundle branch block, ion channel disease, orthostatic intolerance $\beta$ receptor hyperfunction and ECG changes caused by drugs.

diagnosis of cardiogenic chest pain, we use diagnostic criteria of myocarditis and suspected myocarditis from literature reports (16) and our clinical analysis to make the criteria for targeted myocardial enzyme testing for chest pain in children.

\section{Statistical Analysis}

SPSS20.0 was used for statistical analysis. Measurement data are presented as mean \pm standard deviation $(\bar{x} \pm S)$, and count data are expressed as frequency (percentage). The $\chi^{2}$ test was performed to analyze sex distribution and the causes of chest pain. $P<0.05$ was considered statistically significant.

\section{RESULTS}

\section{Demographics}

A total of 7,251 children were included in this study, including 4,293 boys (59.2\%) and 2,958 girls (40.8\%), aged 3-18 years (mean: $12.1 \pm 4.0$ ). Chest pain was more common in school-age children and adolescents. Most patients in the preschool group were girls (55.5\%) while most patients in the school-age and adolescent groups were boys $(60.6 \%, 61.3 \%)$, and this difference in sex distribution was significant $(P<0.01)$.

\section{Pattern of Clinical Visits and Clinical Department for Initial Visit in Children With Chest Pain}

The number of children who saw a doctor for chest pain was increasing each year from 2005 to 2019 (Figure 1). Specifically, 6,312 patients $(87.0 \%)$ visited a clinic, and 926 patients $(12.8 \%)$ went to an emergency room. Only 13 patients $(0.2 \%)$ were admitted to the hospital (Figure 2). At the initial visit, most children saw a pediatric cardiologist $(n=3,477,55.09 \%)$ or a general pediatrician $(n=1,896,30.04 \%)$ (Figure 3).

\section{The History of Past Illness, Family History, Physical Examination, and Concomitant Symptoms in Children With Chest Pain}

None of the children had positive history of past illness. Four patients had a positive family history, including two patients whose fathers had dilated cardiomyopathy, one patient whose sibling had Marfan syndrome, and one patient whose father had died suddenly. Physical examination was positive in 795 children. Among them, 75 patients had pathological heart murmur, including systolic murmur $(n=25)$, diastolic murmur $(n=32)$, accentuated P2 $(n=3)$, splitting of the second heart sound ( $n$ $=12$ ), and continuous machine-like murmur $(n=3)$; 33 had arrhythmia; 62 had a tachycardia; 77 had a bradycardia; 198 patients had fever and bibasilar crackles in both lungs; six had fever and wheezing sounds in both lungs; 327 only had fever; 15 only had wheezing sounds in both lungs; two had hepatomegaly.

Among children with cardiac chest pain $(n=581,8.0 \%)$, the most common concomitant symptom was chest tightness ( $n$ $=389,67.0 \%)$. Among patients with non-cardiac chest pain $(n$ $=6,670,72.0 \%)$, most $(n=4,899,73.4 \%)$ had no concomitant symptoms (Table 4).

\section{The Results of ECG, UCG, Holter Test, and Chest X-Ray}

All 7,251 patients (100\%) underwent ECG, 5,352 (73.8\%) underwent UCG, 128 (1.8\%) underwent Holter test, 1,037 (14.1\%) underwent chest X-ray, and 5,408 (74.6\%) underwent myocardial enzyme testing. 


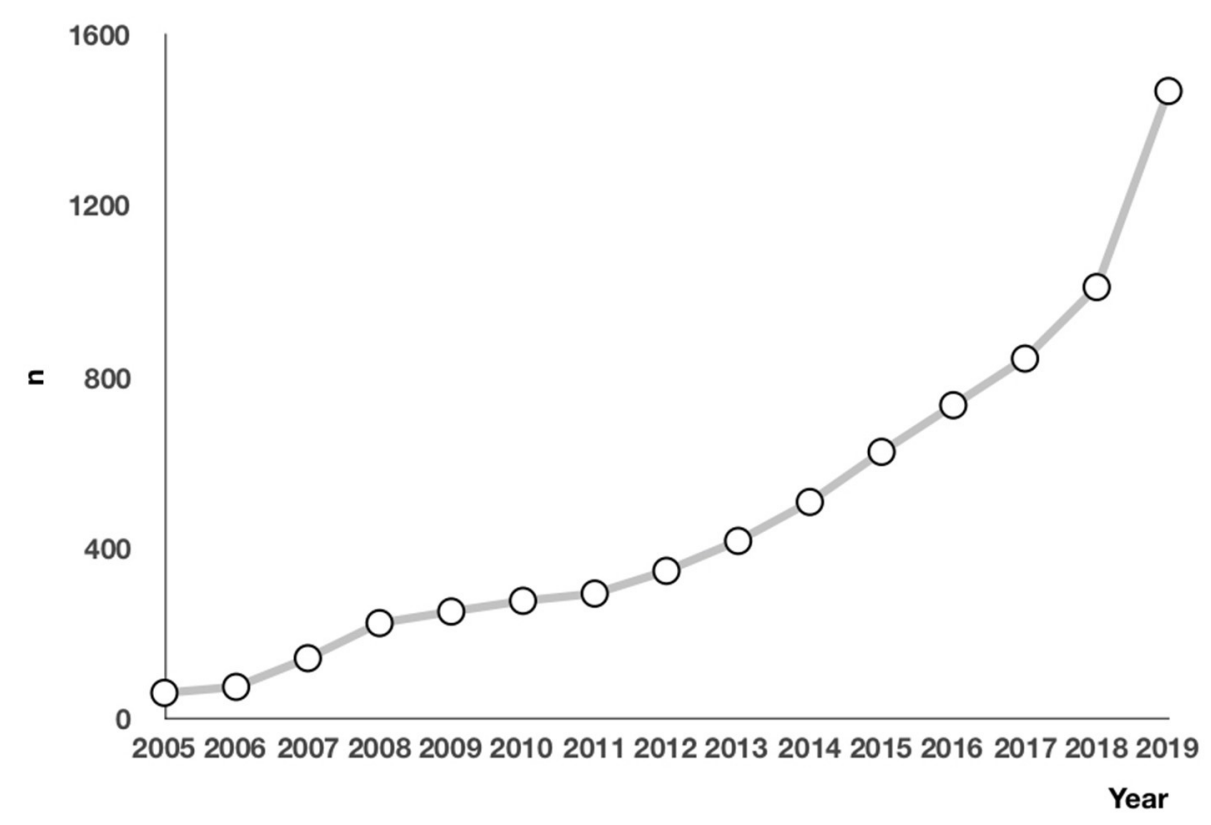

FIGURE 1 | Number of initial evaluations of chest pain in children per year.

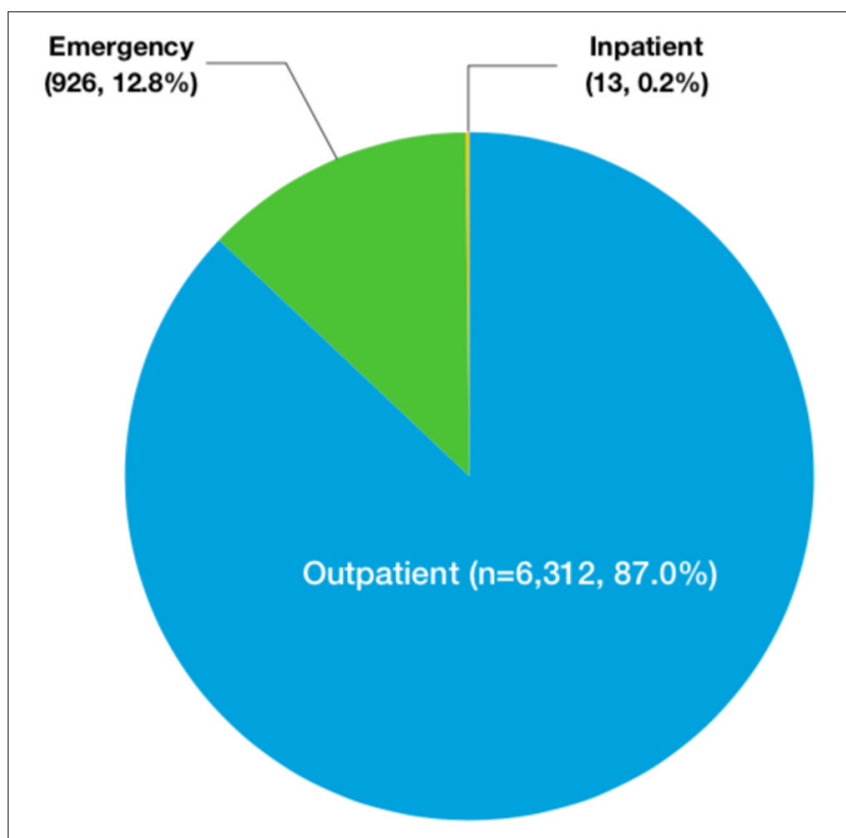

FIGURE 2 | The emergency-department visits of chest pain in children.

ECG was abnormal in 574 of 7,251 children (7.9\%). The most common abnormality was pathological ST-segment or Twave changes ( $n=537,93.6 \%)$, followed by frequent premature ventricular contractions $(n=7,1.2 \%)$, pre-excitation syndrome $(n=10,1.8 \%)$, supraventricular tachycardia $(n=7,1.2 \%)$, deviation of the electrical axis $(n=6,1.0 \%)$, pathological Q wave $(n=6,1.0 \%)$, and left atrial enlargement $(n=1,0.2 \%)$.

UCG was abnormal in 331 of 5,352 children (6.2\%). The abnormality was unrelated to chest pain in 221 of these patients $(66.8 \%)$ and was related to chest pain in 110 of them (33.2\%), including aortic sinus enlargement with aortic sinus aneurysm $(n=3,2.7 \%)$, coronary artery dilation $(n=3,2.7 \%)$, generalized cardiomegaly with reduced left ventricular systolic function $(n=4,3.6 \%)$, pericardial effusion $(n=2,1.8 \%)$, large ventricular septal defect $(n=2$, $1.8 \%)$, severe pulmonary valve stenosis with pulmonary valve regurgitation ( $n=1,0.9 \%)$, quadricuspid aortic valve with aortic sinus aneurysm $(n=1,0.9 \%)$, aortic valve stenosis $(n=3,2.7 \%)$, coronary-pulmonary arterial fistula $(n=4$, $3.6 \%$ ), anomalous origin of the left coronary artery from the pulmonary artery $(n=6,5.5 \%)$, anomalous origin of the right coronary artery from the left sinus $(n=3,2.7 \%)$, pulmonary arterial hypertension $(n=6,5.6 \%)$, and left ventricular enlargement with normal left ventricular systolic function $(n=$ $72,65.5 \%)$.

The Holter test was abnormal in only six of 128 children $(4.7 \%)$, all of whom had frequent premature ventricular contractions. Chest X-ray was abnormal in 643 of 1,037 children (62.0\%). The most common abnormality was patchy opacities in both lungs $(n=225,35.0 \%)$, followed by increased lung markings ( $n=368,57.2 \%)$, pneumothorax $(n=39,6.1 \%)$, and pleural effusion ( $n=11,1.7 \%)$.

Myocardial enzyme tests were abnormal in 453 of 5,408 children (8.4\%). In these 453 patients, $362(80.0 \%)$ had abnormal ECG with normal UCG, 79 (17.4\%) had abnormal ECG and UCG (60 had cardiac dilatation and EF normal or mild decrease, 


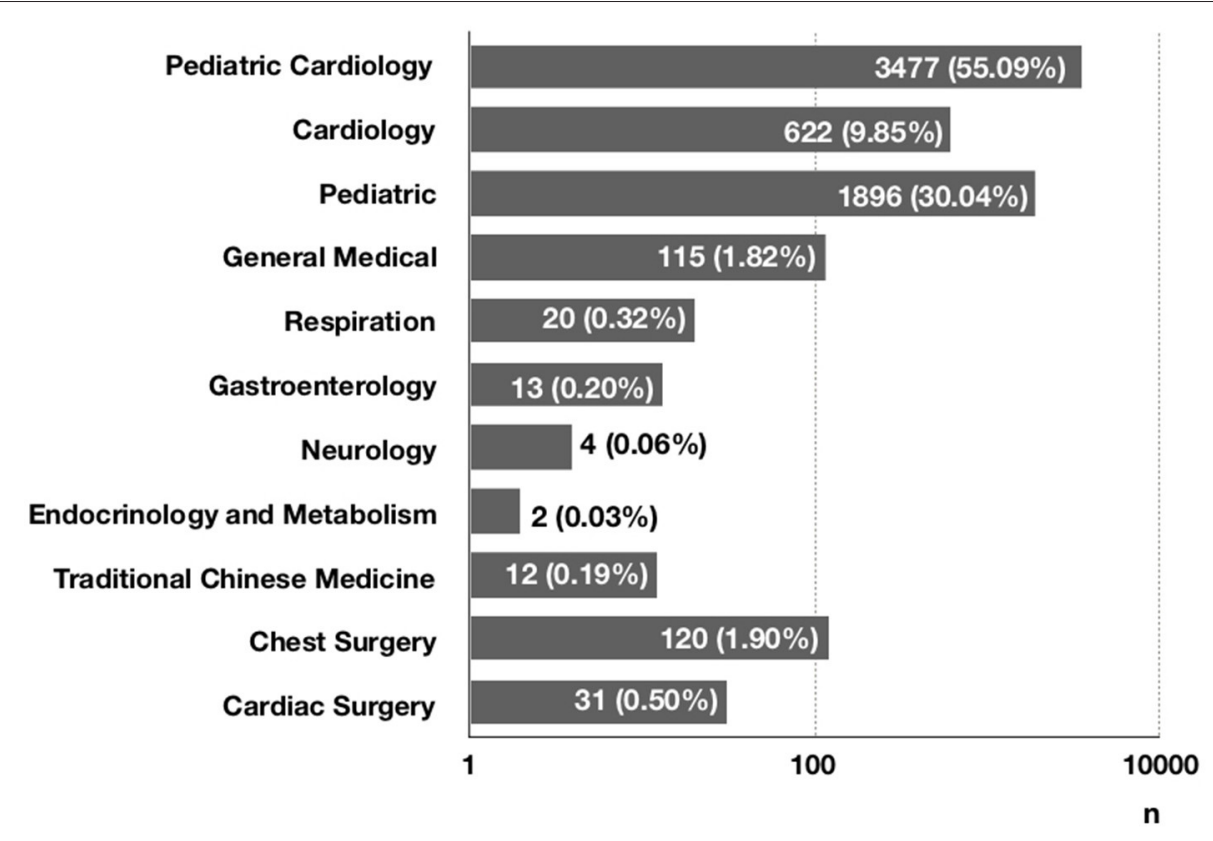

FIGURE 3 | The initial visited departments of chest pain in children.

TABLE 4 | The associated symptoms of chest pain in children.

\begin{tabular}{lc}
\hline Associated symptoms & $\boldsymbol{n}(\mathbf{\%})$ \\
\hline Cardiac-related chest pain & 581 \\
No associated symptoms & $116(20.0 \%)$ \\
Chest oppression & $389(67.0 \%)$ \\
Palpitation & $43(7.4 \%)$ \\
Fatigue & $32(5.5 \%)$ \\
Syncope & $5(0.9 \%)$ \\
Dizzy & $4(0.7 \%)$ \\
Headache & $2(0.3 \%)$ \\
Fever & $4(0.7 \%)$ \\
Respiratory symptoms & $11(1.9 \%)$ \\
Gastrointestinal symptoms & $5(0.9 \%)$ \\
Non-cardiac related chest pain & 6,670 \\
No associated symptoms & $4,899(73.4 \%)$ \\
Chest oppression & $666(10.0 \%)$ \\
Palpitation & $102(1.5 \%)$ \\
Fatigue & $35(0.5 \%)$ \\
Syncope & $14(0.2 \%)$ \\
Dizzy & $46(0.7 \%)$ \\
Jeadache pain & $11(0.2 \%)$ \\
Rever & $2(0.03 \%)$ \\
Gastrointestinal symptoms & $238(3.6 \%)$ \\
\hline & $562(8.4 \%)$ \\
\hline Niratory symptoms & $103(1.5 \%)$ \\
\hline
\end{tabular}

19 had other cardiac disease), 12 (2.6\%) had abnormal UCG (cardiac dilatation and EF normal or mild decrease) with normal ECG.

\section{Diagnostic Results of Chest Pain in Children}

Based on medical history, physical examination, and auxiliary exams, 581 patients $(8.0 \%)$ were diagnosed with cardiac chest pain, and 6,670 patients $(92.0 \%)$ were diagnosed with noncardiac chest pain. Some 3,842 cases $(53.0 \%)$ were idiopathic, $2,109(29.1 \%)$ were related to skeletomuscular diseases, 660 (9.1\%) were related to respiratory diseases, $44(0.6 \%)$ were related to gastrointestinal diseases, $12(0.16 \%)$ were related to mental diseases, and $3(0.04 \%)$ were related to other conditions (Figure 4). The incidence of non-cardiac chest pain was significantly higher in the preschool group and the schoolage group than in the adolescent group (93.5 vs. 93.8 vs. $90.3 \%, P$ $<0.05$ ) (Table 5).

Among the 581 cases of cardiac chest pain, the most common cause was suspected myocarditis ( $n=431,74.2 \%$ ), followed by myocarditis ( $n=82,14.1 \%)$, frequent premature ventricular contractions ( $n=13,2.2 \%)$, pre-excitation syndrome $(n=10$, $1.7 \%)$, supraventricular tachycardia $(n=7,1.2 \%)$, anomalous origin of the left coronary artery $(n=6,1.0 \%)$, pulmonary hypertension $(n=6,1.0 \%)$, dilated cardiomyopathy $(n=$ $4,0.8 \%)$, coronary-pulmonary arterial fistula $(n=4,0.8 \%)$, anomalous origin of the right coronary artery $(n=3,0.5 \%)$, aortic valve stenosis $(n=3,0.5 \%)$, Marfan syndrome $(n=3$, $0.5 \%)$, Kawasaki disease $(n=3,0.5 \%)$, large ventricular septal defect $(n=2,0.3 \%)$, pericardial effusion $(n=2,0.3 \%)$, severe pulmonary valve stenosis with pulmonary valve regurgitation $(n=1,0.2 \%)$, and quadricuspid aortic valve with aortic sinus aneurysm $(n=1,0.2 \%)$ (Figure 5 ).

Six hundred and sixty cases of chest pain were caused by respiratory diseases (Table 6), and 44 cases of chest pain were caused by gastrointestinal diseases (Table 7 ). 


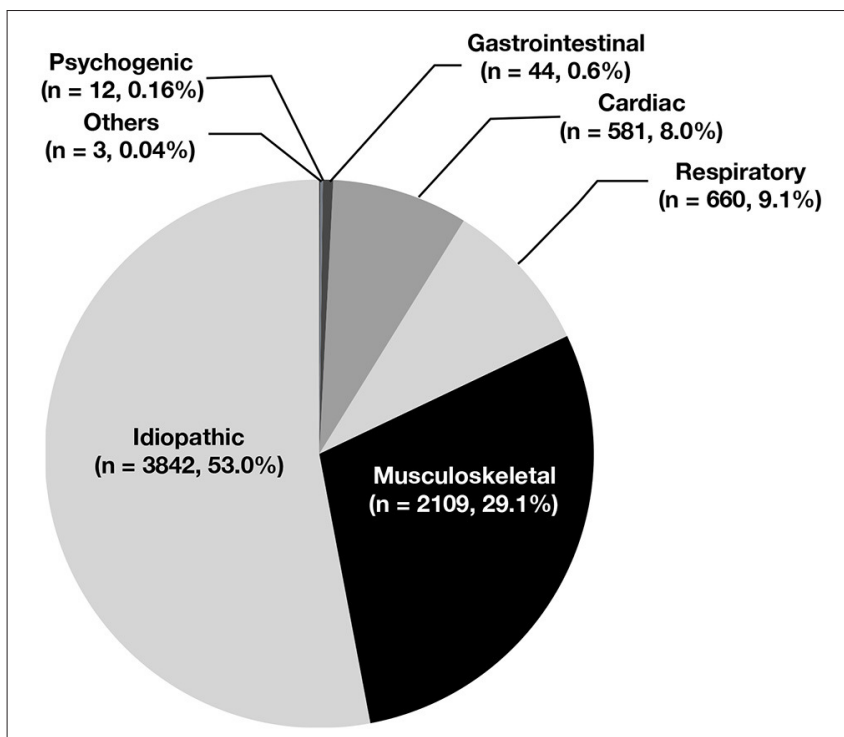

FIGURE 4 | The diagnosis of chest pain in children.

TABLE 5 | The causes of chest pain in children with different ages.

\begin{tabular}{lcccc}
\hline & $\boldsymbol{n}$ & $\begin{array}{c}\mathbf{3} \text { years } \leq \\
\text { age } \leq \mathbf{6} \\
\text { years }\end{array}$ & $\begin{array}{c}\mathbf{6} \text { years }< \\
\text { age } \leq \mathbf{1 2} \\
\text { years }\end{array}$ & $\begin{array}{c}\mathbf{1 2} \text { years }< \\
\text { age } \leq \mathbf{1 8} \\
\text { years }\end{array}$ \\
\hline Total & 7,251 & 784 & 2,779 & 3,688 \\
Cardiac & $581(8.0 \%)$ & $51(6.5 \%)$ & $171(6.2 \%)$ & $359(9.7 \%)$ \\
Non-cardiac & 6,670 & $733(93.5 \%)$ & 2608 & 3,329 \\
X2 & $(92.0 \%)$ & & $(93.8 \%)$ & $(90.3 \%)$ \\
$P$ & & 8.096 & 27.011 & \\
\hline
\end{tabular}

\# stands for $P<0.05$ contrast to 12 years $<$ age $\leq 18$ years group.

Among the 2,109 cases of chest pain due to skeletomuscular diseases, the most common cause was precordial catch syndrome ( $n=1,308,62.1 \%)$, followed by slipping rib syndrome $(n=787$, $37.3 \%)$, costochondritis $(n=7,0.3 \%)$, trauma $(n=5,0.2 \%)$, and straight-back syndrome $(n=2,0.1 \%)$. Among the 12 cases of chest pain due to mental diseases, the causes included anxiety $(n=5)$, depression $(n=4)$, and hysteria-like attacks $(n=3)$. Meanwhile, patients with mental diseases-related chest pain were all adolescents. Among the three cases of chest pain due to other disorders, the causes included breast mass $(n=1)$, dysmenorrhea $(n=1)$, and poor wound healing $(n=1)$.

\section{Diagnostic Procedures and Criteria for Targeted Myocardial Enzyme Testing for Chest Pain in Children}

Based on the diagnostic procedures for chest pain in children from literature reports and our analysis, we recommend that for children with chest pain, clinicians collect detailed information about the history of the present illness, past medical history, and family history and perform a comprehensive physical examination to distinguish cardiac from non-cardiac chest pain. Patients with potential cardiac chest pain should be referred to a pediatric cardiologist and undergo ECG or ECG plus UCG if they have a pathological heart murmur or hypoxemia, respectively. Patients should undergo myocardial enzyme testing if they have abnormal ECG plus normal UCG or abnormal/normal ECG plus abnormal UCG, suggesting cardiomegaly with normal or mildly reduced left ventricular systolic function. Myocardial enzyme testing is not recommended for patients with normal ECG plus normal UCG or normal ECG plus abnormal UCG, suggesting other cardiac malformations (Figure 6).

Children with non-cardiac chest pain should see an appropriate specialist based on medical history and physical examination. Patients may continue to receive treatment or follow up if the chest pain alleviates or resolves after treatment, or they can visit a pediatric cardiologist for evaluation if the chest pain does not improve after treatment (Figure 6).

For these children with chest pain, if they were evaluated by this flow chart, 580 patients will be referred to a pediatric cardiologist, and then 75 will undergo ECG and UCG examination, 505 will undergo ECG examination only. Through analyzing these 580 patients' ECG or UCG, 512 will undergo myocardial enzyme testing. Based on the diagnostic procedures, 509 patients could be diagnosed as cardiac chest pain, 72 patients with cardiac chest pain would be missed. So, the positive diagnostic value for cardiac chest pain of this flow chart was $87.6 \%$. Meanwhile, through the diagnostic procedures, pediatric cardiac specialist outpatient visit rate and unnecessary auxiliary examination significantly reduced, respectively.

\section{DISCUSSION}

Chest pain is a common symptom. With the improvement of living standards and attention to quality of life, more children are seeing a doctor for chest pain. In 2011, Saleeb et al. (19) analyzed the clinical data of children who visited Boston Children's Hospital for chest pain between 2000 and 2009 and found that the number of children who saw a doctor for chest pain increased each year. This study showed a similar trend at our hospital between 2005 and 2019, suggesting that parents are paying more attention to their children's symptoms and that many parents believe that chest pain in children is cardiac and life-threatening, due to a lack of knowledge in the common causes of chest pain in children. As a result, they often take their children to an emergency or a pediatric cardiologist. In fact, most cases of chest pain in children are non-cardiac and benign (3-7, 9$11,15,18,19)$. In 2011, Saleeb et al. (19) retrospectively analyzed the pattern of visits, causes, and mortality of chest pain in 3,700 children and found that $18 \%$ of the patients visited an emergency room and that $99 \%$ of the cases were non-cardiac. During the 4.4years follow-up, only three patients died of non-cardiac diseases. This indicates that mortality is low in children with chest pain, with few cardiac deaths. In 2020, Gesuete et al. analyzed the clinical data of 761 children who went to an emergency for chest pain and found that only $1 \%$ of the cases were cardiac (11). Our study showed that $92.0 \%$ of the cases were non-cardiac, 
Quadricuspid aortic valve with sinus of Valsalva aneurysm ( $n=1,0.2 \%)$

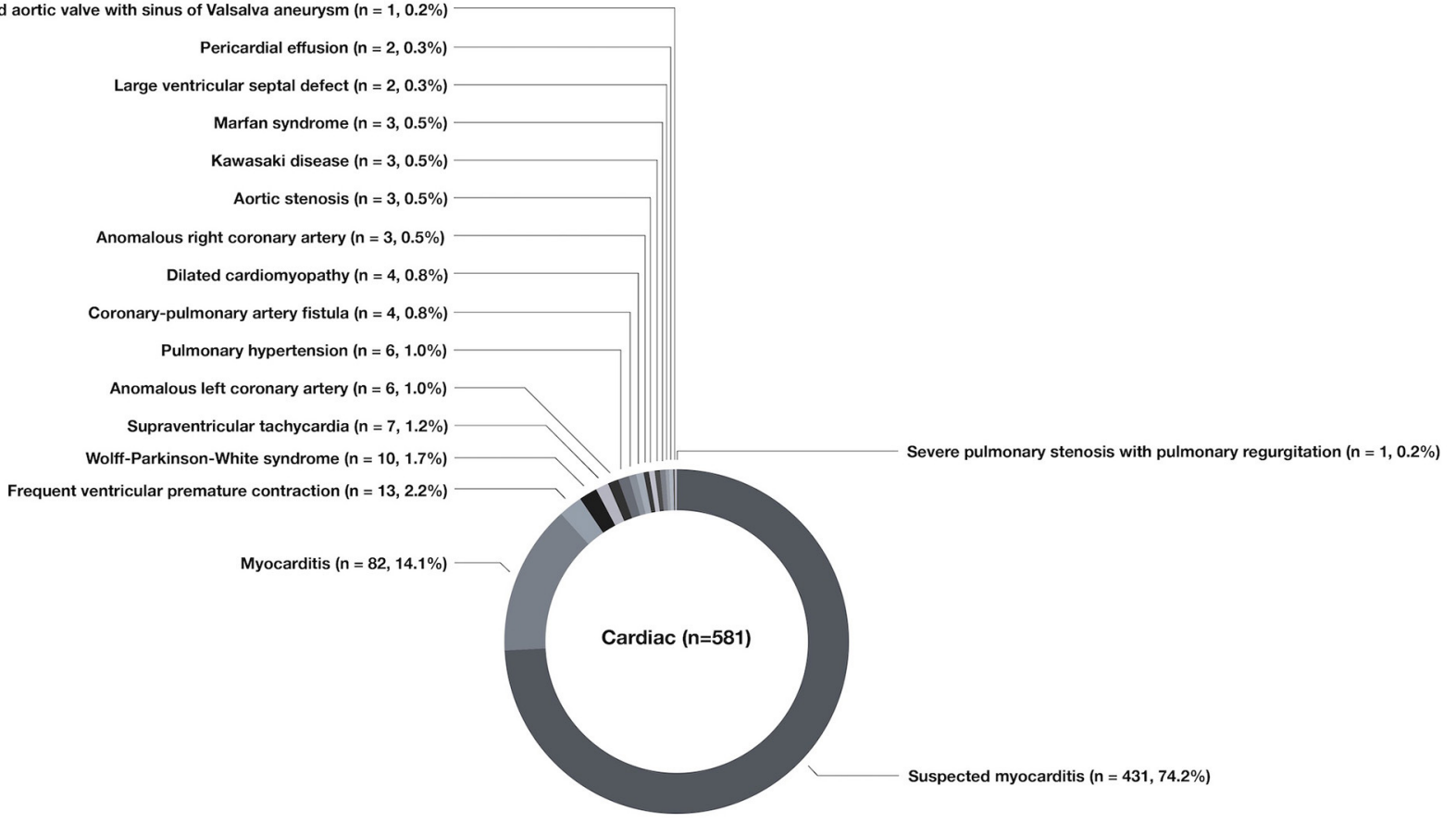

FIGURE 5 | The cardiac diseases causing chest pain in children.

TABLE 6 | The respiratory diseases causing chest pain in children.

\begin{tabular}{lc}
\hline Respiratory diseases $(\boldsymbol{n}=\mathbf{6 6 0})$ & $\boldsymbol{n}(\mathbf{\%})$ \\
\hline Bronchitis & $357(54.1 \%)$ \\
Pneumonia & $224(33.9 \%)$ \\
Pneumothorax & $38(5.7 \%)$ \\
Asthma & $15(2.2 \%)$ \\
Pleuritis & $10(1.5 \%)$ \\
Acute asthmatic bronchitis & $6(0.9 \%)$ \\
Chronic cough & $5(0.7 \%)$ \\
Emphysema & $1(0.2 \%)$ \\
Mediastinal emphysema & $1(0.2 \%)$ \\
Pulmonary bullae & $1(0.2 \%)$ \\
Pulmonary tuberculosis & $1(0.2 \%)$ \\
Pleural effusion & $1(0.2 \%)$ \\
\hline
\end{tabular}

$12.8 \%$ of the patients went to an emergency, and $55.09 \%$ saw a pediatric cardiologist, suggesting that most parents believe that their children's chest pain is critical and has a cardiac cause. Therefore, it is important to educate the public about chest pain in children, investigate the causes of chest pain in children, and develop standard diagnostic procedures, in order to reduce resource use.

Chest pain in children may be cardiac or non-cardiac (5, 16). The causes of cardiac chest pain include (1) coronary artery diseases; (2) arrhythmia; (3) inflammatory diseases; (4) structural abnormalities $(5,16)$. The causes of non-cardiac
TABLE 7 | The gastrointestinal diseases causing chest pain in children.

\begin{tabular}{lc}
\hline Gastrointestinal diseases $(\boldsymbol{n}=\mathbf{4 4 )}$ & $\boldsymbol{n}(\mathbf{\%})$ \\
\hline Gastritis & $28(63.6 \%)$ \\
Gastroesophageal reflux & $13(29.5 \%)$ \\
Esophagitis & $1(2.3 \%)$ \\
Esophageal hiatal hernia & $1(2.3 \%)$ \\
Constipation & $1(2.3 \%)$
\end{tabular}

chest pain include respiratory diseases, gastrointestinal diseases, skeletomuscular diseases, mental diseases, and idiopathic factors. In 2011, Saleeb et al. retrospectively analyzed the causes of chest pain in 3,700 children and found that $52 \%$ of the cases were idiopathic, $36 \%$ were related to skeletomuscular diseases, $7 \%$ were related to gastrointestinal diseases, $1 \%$ were related to mental disorder, and only $1 \%$ were cardiac. The most common cause of cardiac chest pain was inflammatory disease, such as pericarditis and myocarditis (19).

This is the first large study to investigate the causes of chest pain in Chinese children. We analyzed the causes of chest pain in 7,251 children who visited our hospital during a 15-years period and found that $53.0 \%$ of the cases were idiopathic, $29.1 \%$ were related to skeletomuscular diseases, $9.1 \%$ were related to respiratory diseases, $8.0 \%$ were cardiac, $0.6 \%$ were related to gastrointestinal diseases, $0.16 \%$ were related to mental diseases, and $0.04 \%$ were related to other conditions. These data indicate that idiopathic chest pain was the most common cause of chest pain in Chinese children, which is consistent with foreign reports. 


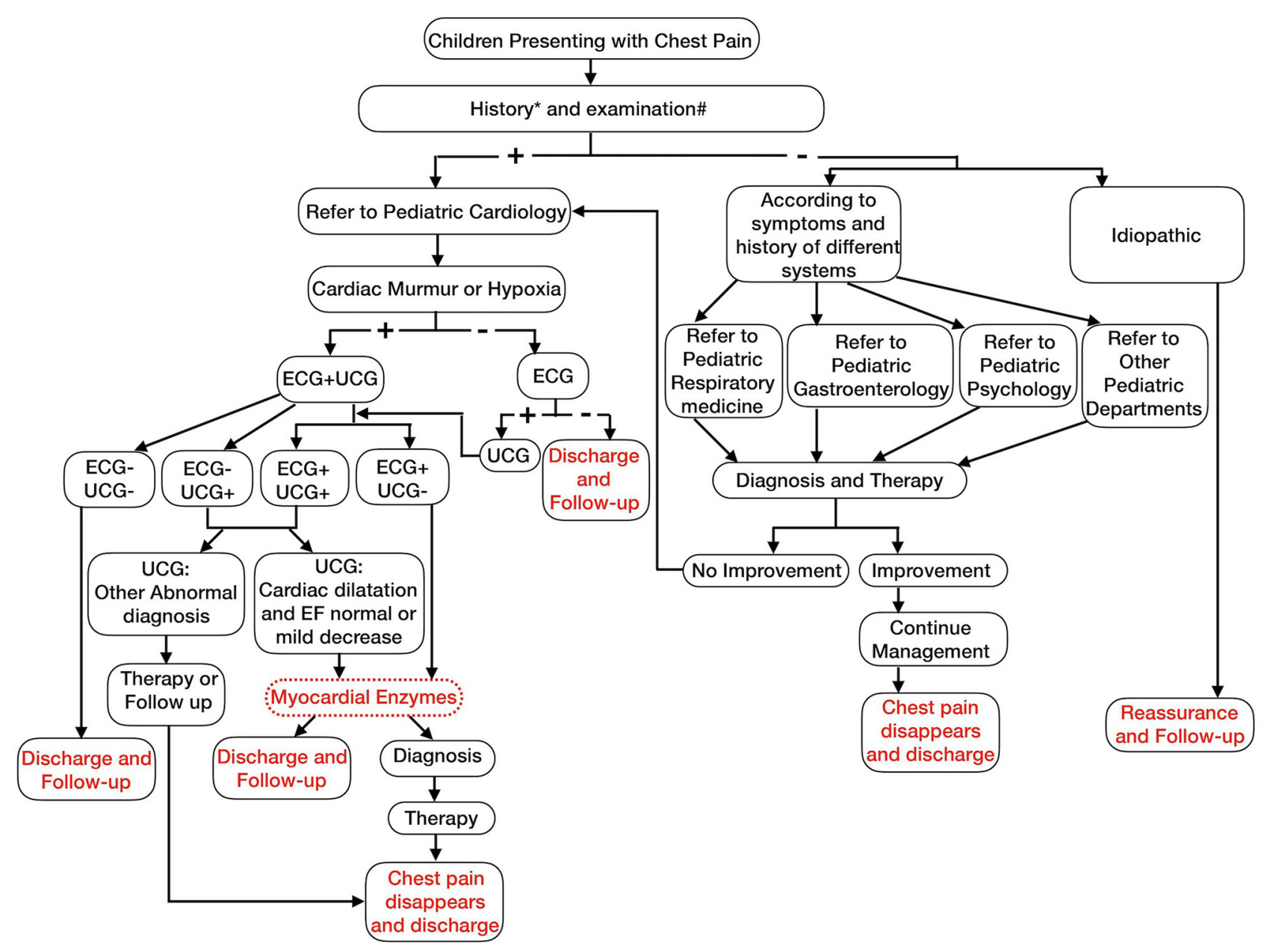

FIGURE 6 | Chest pain management algorithm (History* includes fever, cough, stomach ache associated with chest pain, abnormal mental state, palpitations associated with chest pains, syncope during activity, chest pain during exercise, history of cardiac surgery or interventions, complex congenital heart defects, Marfan syndrome, children with on specific cardiac drugs heart transplant, history of Kawasaki disease, history of drug abuse, significant family history of arrhythmias, sudden death in young adulthood, genetic disorders linked with arrhythmias e.g., Long QT syndromes or Brugada syndrome, first-degree relatives have familial hypercholesterolemia, cardiomyopathy and pulmonary hypertension; Examination\# include fever, abnormal breath sounds, pathologic murmur, abnormal heart sounds, hypoxia, peripheral edema, hepatomegaly, significant tachycardia, or irregular rhythm).

In our study, we also found that skeletomuscular-related chest pain was the second reason. Idiopathic and skeletomuscularrelated chest pain always were not life threatening, so for most children with chest pain, their parents or guardian could not be scared and go to emergency immediately. Meanwhile, $0.16 \%$ were related to mental diseases in our study. Through analyzing their ages, we found that they were all adolescents. In adolescence, they might have study pressure, social pressure and the influence of puberty hormone level, for releasing the pressure, they complained chest pain. So for adolescents with chest pain, the parents or guardian should give them more care, and this can relieve their chest pain.

At their initial visit, most patients with chest pain see a pediatric cardiologist. Moreover, parents often request cardiac auxiliary exams to exclude cardiac chest pain. As a result, most children with chest pain undergo various unnecessary auxiliary exams. Therefore, it is important to develop diagnostic procedures for chest pain in children in order to reduce resource use. Collins et al. (12), Harahsheh et al. (13), and Etuwewe et al. (14) proposed evaluation methods and procedures for chest pain in children (12-14). The researchers stressed the importance of detailed medical history and physical examination to determine the potential cause of chest pain, followed by necessary exams. The researchers, however, did not describe how to choose the auxiliary exams. In 2011, Friedman et al. (15) reported that they developed standard clinical evaluation and treatment procedures for chest pain in children and applied these procedures in children who saw a pediatric cardiologist at Boston Children's Hospital for chest pain between 2000 and 2009. The researchers believed that patients with exercise-related chest pain or positive family history should undergo comprehensive cardiac evaluation, including UCG, for further diagnosis. With these procedures, 
UCG usage was reduced by $20 \%$ without missing any case of cardiac chest pain, thereby reducing resource use and the costs to the families.

Myocardial enzyme testing is widely used in clinical practice to help diagnose heart conditions. In China, many doctors order myocardial enzyme tests at the initial visit to exclude myocarditis or suspected myocarditis in children with chest pain. However, the test result is normal in most cases. In 2020, the Subspecialty Group of Cardiology, Society of Pediatrics, Chinese Medical Association, issued recommendations for the diagnosis of myocarditis in children and clearly stated the diagnostic criteria for myocarditis and suspected myocarditis in children (16). No past studies have investigated how to choose the myocardial enzyme test and reduce their use without missing any case of cardiac chest pain in children. The present study showed that for children with cardiac chest pain, the most common concomitant symptom was chest tightness $(67.0 \%)$ while most patients with non-cardiac chest pain had no concomitant symptoms, and respiratory or gastrointestinal symptoms were the main concomitant symptoms (if any). Therefore, concomitant symptoms may help evaluate the potential cause of chest pain in children, followed by auxiliary exams. In this study, among the 7,251 children with chest pain, myocardial enzyme tests were abnormal in only 453 of 5,408 patients $(8.4 \%)$. In these 453 patients, $362(80.0 \%)$ had abnormal ECG with normal UCG, 79 (17.4\%) had abnormal ECG and UCG (60 had cardiac dilatation and EF normal or mild decrease, 19 had other cardiac disease), 12 (2.6\%) had abnormal UCG (cardiac dilatation and EF normal or mild decrease) with normal ECG. These results suggested that myocardial enzyme testing is unnecessary in patients without symptoms or signs of myocarditis or suspected myocarditis during the initial evaluation. Such an approach would help reduce resource use and unnecessary treatment.

This is the first study to develop diagnostic procedures and criteria for targeted myocardial enzyme testing in children with chest pain. Through analyzing the clinical data of 7,251 children who visited our hospital for chest pain during a 15-years period and referencing the diagnostic procedures from literature reports, we recommend that clinicians evaluate the potential cause of chest pain based on the history of the present illness, past medical history, family history, and physical examination and refer patients to an appropriate specialist. Patients with potential cardiac chest pain should undergo ECG or ECG plus UCG if they have a pathological heart murmur or hypoxemia, respectively. Patients should undergo myocardial enzyme testing if they have abnormal ECG plus normal UCG or normal/abnormal ECG plus abnormal UCG, suggesting cardiomegaly with normal or mildly reduced left ventricular systolic function. Patients with non-cardiac chest pain may continue to receive treatment or follow-up if the chest pain alleviates or resolves after treatment, or they should be referred to a pediatric cardiologist for evaluation to exclude heart conditions if the chest pain does not improve after treatment. Patients with idiopathic chest pain may receive counseling and follow-up. These procedures reduce visits and unnecessary myocardial enzyme tests, thereby reducing resource use.
There were some limitations in our study. Firstly, this was a retrospective study in one single center, so the relevant data were affected by the hospital size, first line specialty, patients' source and other aspects. The relevant results may not perfectly represent all the children patients in China. However, as one of the largest cardiovascular hospitals in China and even in Asia, our study involved the largest number of children with chest pain in China and the screening for children's heart might also be the most comprehensive, our results showed that there was no clear correlation between chest pain and cardiovascular disease in children, so we believe this result should be correct and reliable. Secondly, since this retrospective study spans 15 years, the diagnostic criteria and ICD codes of some diseases have changed during this period, which might have some impact on our research results. In order to avoid this kind of influence, we verified the diagnosis not only by diagnostic coding but also by analyzing the results of auxiliary exams. Furthermore, as the diagnostic procedures we proposed here were designed based on the results of retrospective study, prospective studies in future should be conducted to further confirm the efficacy, feasibility and superiority of the diagnostic procedure. In the future, we will conduct a large multicenter clinical study to further investigate the cause of chest pain in Chinese children, and explore a non-invasive and effective diagnostic method for children with chest pain. Meanwhile, we will conduct a prospective study to investigate the efficacy of our diagnostic procedures and criteria for targeted myocardial enzyme testing in children with chest pain. Based on the above, it can be predicted that an economic and effective diagnostic procedure for chest pain in Chinese children will be established.

\section{DATA AVAILABILITY STATEMENT}

The original contributions presented in the study are included in the article/supplementary material, further inquiries can be directed to the corresponding author/s.

\section{ETHICS STATEMENT}

The studies involving human participants were reviewed and approved by The Ethics Committee of Beijing Anzhen Hospital, Capital Medical University. Written informed consent to participate in this study was provided by the participants' legal guardian/next of kin.

\section{AUTHOR'S NOTE}

Chest pain is a common complaint at paediatric clinics, accounting for $\sim 6$ per 1000 visits at paediatric emergency room. Most parents bring their child who has chest pain to an emergency room or see a paediatric cardiologist for comprehensive cardiac exams, which affects the child's life and schoolwork and increases the costs to their families. To date, no large studies have been conducted to investigate the causes of chest pain in Chinese children and no studies have investigated targeted cardiac enzyme testing for the diagnosis of 
chest pain in children. Our study shows that chest pain is more common in school-age children and adolescents. For Chinese children with chest pain, the most common cause is idiopathic chest pain. Few cases are cardiac chest pain. Patients with potential cardiac chest pain should undergo ECG or ECG plus UCG if they have a pathological heart murmur or hypoxemia, respectively. Patients should undergo myocardial enzyme testing if they have abnormal ECG plus normal UCG or abnormal ECG plus abnormal UCG (UCG suggests cardiomegaly with normal or mildly reduced left ventricular systolic function). This approach would reduce resource use and the costs to the families.

\section{REFERENCES}

1. Selbst SM. Consultation with the specialist. Chest pain in children. Pediatr Rev. (1997) 18:169-73. doi: 10.1542/pir.18-5-169

2. Andrew WB, Julian F. How to evaluate a child with chest pain. Curr Paediatr. (2004) 14:64-70. doi: 10.1016/j.cupe.2003.09.003

3. Tunaoglu FS, Olgunturk R, Akcabay S, Oguz D, Gucuyener K, Demirsoy S. Chest pain in children referred to a cardiology clinic. Pediatr Cardiol. (1995) 16:69-72. doi: 10.1007/BF00796820

4. Talner NS, Carboni MP. Chest pain in the adolescent and young adult. Cardiol Rev. (2000) 8:49-56. doi: 10.1097/00045415-200008010-00009

5. Cava JR, Sayger PL. Chest pain in children and adolescents. Pediatr Clin North Am. (2004) 51:1553-1568, viii. doi: 10.1016/j.pcl.2004.07.002

6. Danduran MJ, Earing MG, Sheridan DC, Ewalt LA, Frommelt PC. Chest pain: characteristics of children/adolescents. Pediatr Cardiol. (2008) 29:775-81. doi: 10.1007/s00246-008-9200-9

7. Lin $\mathrm{CH}$, Lin WC, Ho YJ, Chang JS. Children with chest pain visiting the emergency department. Pediatr Neonatol. (2008) 49:26-9. doi: 10.1016/S1875-9572(08)60007-8

8. Thull-Freedman J. Evaluation of chest pain in the pediatric patient. Med Clin North Am. (2010) 94:327-47. doi: 10.1016/j.mcna.2010.01.004

9. Drossner DM, Hirsh DA, Sturm JJ, Mahle WT, Goo DJ, Massey R, et al. Cardiac disease in pediatric patients presenting to a pediatric ED with chest pain. Am J Emerg Med. (2011) 29:632-8. doi: 10.1016/j.ajem.2010.01.011

10. Kane DA, Fulton DR, Saleeb S, Zhou J, Lock JE, Geggel RL. Needles in hay: chest pain as the presenting symptom in children with serious underlying cardiac pathology. Congenit Heart Dis. (2010) 5:366-73. doi: 10.1111/j.1747-0803.2010.00436.x

11. Gesuete V, Fregolent D, Contorno S, Tamaro G, Barbi E, Cozzi G. Followup study of patients admitted to the pediatric emergency department for chest pain. Eur J Pediatr. (2020) 179:303-8. doi: 10.1007/s00431-01903495-5

12. Collins SA, Griksaitis MJ, Legg JP. 15-minute consultation: a structured approach to the assessment of chest pain in a child. Arch Dis Child Educ Pract Ed. (2014) 99:122-6. doi: 10.1136/archdischild-2013-303919

13. Harahsheh AS, O’Byrne ML, Pastor B, Graham DA, Fulton DR. Pediatric chest pain-low-probability referral: a multi-institutional analysis from standardized

\section{AUTHOR CONTRIBUTIONS}

LC, HD, XL, ZY, MJ, KS, and MJ made substantial contributions to the conception or design of the work or the acquisition, analysis or interpretation of data. LC drafted the work. HD and MJ revised the work critically for important intellectual content. All authors read and approved the final manuscript.

\section{FUNDING}

This study was supported by Youth Clinical Research Project of Peking University First Hospital (No. 2019CR02).

clinical assessment and management plans (scamps $($ )), the pediatric health information systems database, and the national ambulatory medical care survey. Clin Pediatr. (2017) 56:1201-8. doi: 10.1177/0009922816684605

14. Etuwewe BB. Assessing chest pain in children. Paediatr Child Health. (2018) 28:348-50. doi: 10.1016/j.paed.2018.05.004

15. Friedman KG, Kane DA, Rathod RH, Renaud A, Farias M, Geggel $\mathrm{R}$, et al. Management of pediatric chest pain using a standardized assessment and management plan. Pediatrics. (2011) 128:239-45. doi: 10.1542/peds.2011-0141

16. Subspecialty Group of Cardiology, the Society of Pediatrics, Chinese Medical Association; Collaborating Group of Myocarditis, the Subspecialty Group of Cardiology, the Society of Pediatrics, Chinese Medical Association; Editorial Board, Chinese Journal of Pediatrics; Pediatric Cardiology Committee, Chinese College of Cardiovascular Physicians, Chinese Medical Doctor Association. Diagnostic recommendation for myocarditis in children (version 2018). Zhonghua Er Ke Za Zhi. (2019) 57:87-9. doi: 10.3760/cma.j.issn.0578-1310.2019.02.004

17. Friedman KG, Alexander ME. Chest pain and syncope in children: a practical approach to the diagnosis of cardiac disease. J Pediatr. (2013) 163:896-901. doi: 10.1016/j.jpeds.2013.05.001

18. Pantell RH, Goodman BW. Adolescent chest pain: a prospective study. Pediatrics. (1983) 71:881-7.

19. Saleeb SF, Li WY, Warren SZ, Lock JE. Effectiveness of screening for life-threatening chest pain in children. Pediatrics. (2011) 128:e1062-8. doi: $10.1542 /$ peds.2011-0408

Conflict of Interest: The authors declare that the research was conducted in the absence of any commercial or financial relationships that could be construed as a potential conflict of interest.

Copyright (C) 2021 Chen, Duan, Li, Yang, Jiao, Sun and Jin. This is an open-access article distributed under the terms of the Creative Commons Attribution License (CC $B Y)$. The use, distribution or reproduction in other forums is permitted, provided the original author(s) and the copyright owner(s) are credited and that the original publication in this journal is cited, in accordance with accepted academic practice. No use, distribution or reproduction is permitted which does not comply with these terms. 